\title{
From Plasmodium vivax outbreak to elimination: lessons learnt from a retrospective analysis of data from Guantang
}

Guo-Jing Yang ${ }^{1,2,3^{*}}$ (D, Ying Liu ${ }^{4}$, Le-Yuan Shang ${ }^{4}$, Hong-Wei Zhang ${ }^{4}$, Xiao-Nong Zhou ${ }^{5,6}$, Melissa A. Penny ${ }^{1,2}$ and Thomas A. Smith ${ }^{1,2}$

\begin{abstract}
Background: Malaria was once a serious public health problem in China, with Plasmodium vivax the major species responsible for more than $90 \%$ of local transmission. Following significant integrated malaria control and elimination programmes, malaria burden declined, and since 2017 China has not recorded any indigenous case. To understand the historical malaria transmission patterns and epidemic characteristics in China and insights useful to guide P. vivax malaria control and elimination elsewhere, a retrospective study was carried out.

Methods: Historical data from a pilot study conducted in Guantang, Luyi in central China from 1971-1995, were digitized. The data included monthly numbers of reported cases, febrile cases, parasite carriage rates, the neonatal infection rate, and entomological data regarding Anopheles sinensis.

Results: Following 25 years of continuous integrated malaria control activities, malaria incidence in Guantang decreased from 4,333 cases per 10,000 in 1970 before integrated implementation to 0.23 cases per 10,000 in 1991, and no cases in 1992-1995. Some fluctuations in incidence were observed between 1977 and 1981. During the period parasite rates, antibody levels and the neonatal infection rate also decreased. The pattern of seasonality confirmed that $P$. vivax in Henan Province was primarily of the long incubation type (temperate) during non-transmission period. The findings retrospectively provide a scientific basis for the implementation of mass campaigns of liver stage hypnozoite clearance. Entomological studies indicated that An. sinensis was the only vector, and it preferred bovine to human hosts, predominantly biting and resting outdoors. Mosquito densities declined between 1971 and 1984 .

Conclusion: The integrated malaria control approach in Guantang effectively controlled malaria and achieved elimination. Analysis of the effectiveness of the programme can provide guidance to other regions or countries with similar ecological settings aiming to move from malaria control to elimination. There is a potential challenge in the maintenance of non-transmission status owing to imported cases and the long dormancy of liver stage hypnozoites.
\end{abstract}

Keywords: Plasmodium vivax, Central China, Pattern, Elimination

*Correspondence: guojingyang@hotmail.com

1 Swiss Tropical and Public Health Institute, Socinstrasse 57, 4002 Basel, Switzerland

Full list of author information is available at the end of the article

\section{Background}

Malaria was once a serious public health problem in the People's Republic of China (P. R. China) [1] with Henan province, located in the centre of the country, characteristic of endemic zones. Henan is in the transition zone from subtropical to warm temperate, and the history of

c) The Author(s) 2020. This article is licensed under a Creative Commons Attribution 4.0 International License, which permits use, sharing, adaptation, distribution and reproduction in any medium or format, as long as you give appropriate credit to the original author(s) and the source, provide a link to the Creative Commons licence, and indicate if changes were made. The images or other third party material in this article are included in the article's Creative Commons licence, unless indicated otherwise in a credit line to the material. If material is not included in the article's Creative Commons licence and your intended use is not permitted by statutory regulation or exceeds the permitted use, you will need to obtain permission directly from the copyright holder. To view a copy of this licence, visit http://creativeco mmons.org/licenses/by/4.0/. The Creative Commons Public Domain Dedication waiver (http://creativecommons.org/publicdomain/ zero/1.0/) applies to the data made available in this article, unless otherwise stated in a credit line to the data. 
malaria there can be traced back to 4000 years ago [1,2]. Malaria used to be widespread in Henan with an unstable pattern of transmission, and with Plasmodium vivax accounting for more than $90 \%$ of malaria cases [3, 4]. Following the founding of the P. R. China, a notification system for infectious diseases was established. A total of 39 infectious diseases are notifiable by law and categorized as $\mathrm{A}, \mathrm{B}$ or $\mathrm{C}$ diseases with malaria listed as a class B infection from 1956 onwards [5, 6]. Malaria control became part of a systematically organized disease prevention and control system with policy implementation and guidance organized vertically (Fig. 1). After the implementation of integrated malaria control and elimination programmes, the disease burden sharply declined, with no indigenous cases announced in 2017 at a national level [7].

At the national level, the post-1949 history of malaria in China can be primarily grouped into five phases: (i) transmission unknown (1949-1959); (ii) outbreak and pandemic transmission (1960-1979); (iii) decline with sporadic cases (1980-1999); (iv) low transmission with re-emergence in central China (2000-2009); and, (v) the elimination phase (2010-2020) [7, 8]. In parallel with these phases at national level, malaria control in Henan experienced five stages: (i) investigation and target intervention (1950-1962); (ii) disease control (1963-1984); (iii) pre-elimination (1985-1994); (iv) consolidation (1995-2009); and (v) elimination (2010-2020) [1, 3, 4]. (Fig. 2).

The former Ministry of Health, along with 13 additional ministries, issued the National Malaria Elimination Action Plan (2010-2020) (NMEAP), with the objective of eliminating indigenous malaria in non-border regions before the end of 2015 and eliminating the disease nationwide before the end of 2020 [7]. Through decades of continuous malaria control activities, no indigenous malaria case has been reported since 2012 in Henan Province, 3 years ahead of the NMEAP target [9].

To understand the dynamics of this successful programme, data from one area in Henan that was monitored closely throughout this progression from control to elimination have been extracted. This paper summarizes the experiences and lessons of the programme, which

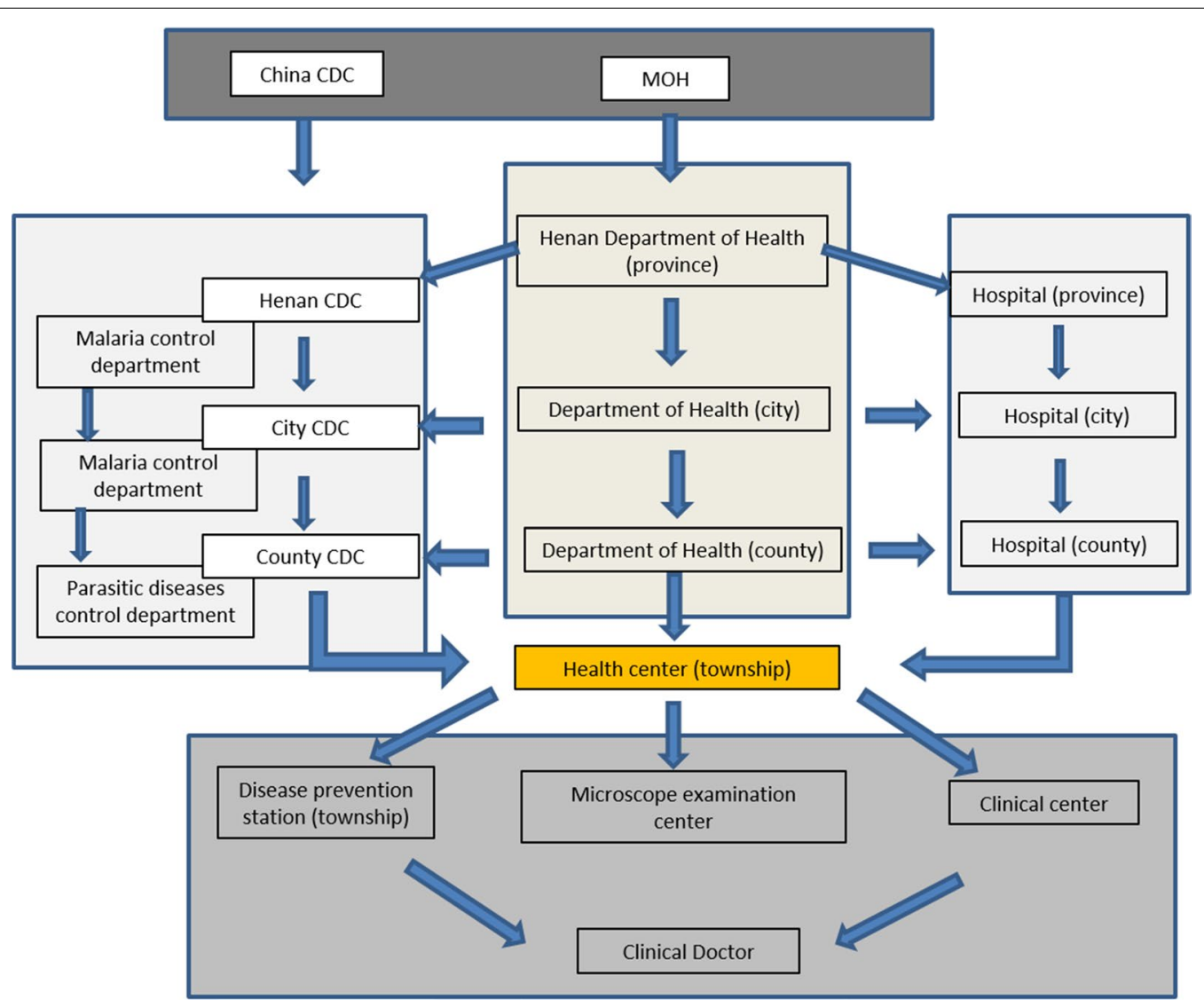

Fig. 1 Malaria vertical prevention and control system with policy implementation and guidance: the malaria prevention and control network consists of professional prevention and control centers, government departments, and medical and health institutions at all levels. Under the direct leadership of the government departments at the same level, they undertake corresponding tasks 


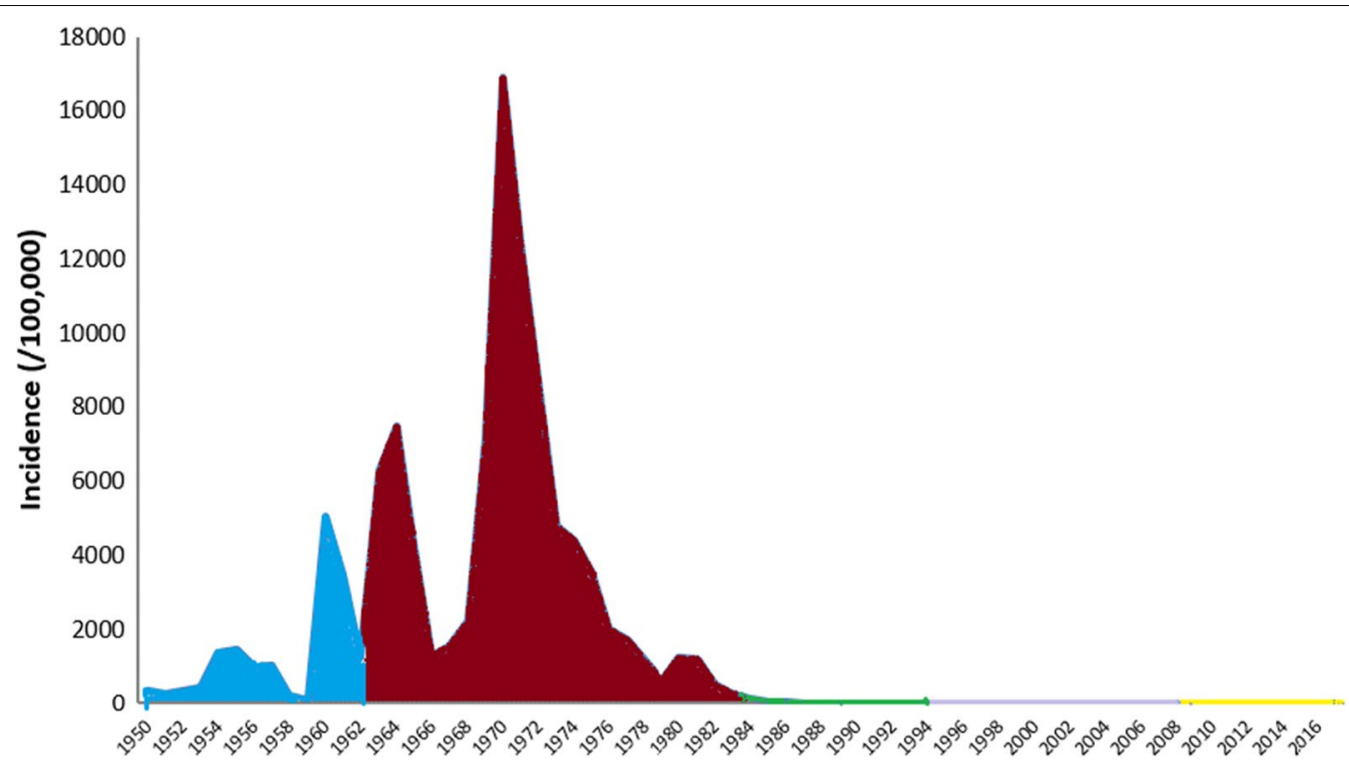

Fig. 2 Malaria control history in Henan province between 1950 to 2017: (i) investigation and target intervention (1950-1962, blue); (ii) disease control (1963-1984, red); (iii) pre-elimination (1985-1994, green); (iv) consolidation (1995-2009, purple); and (v) elimination (2010-2020, yellow)

could provide insights to other regions or countries with similar ecological settings aiming to move from malaria control to elimination.

\section{Methods}

Historical data from a pilot study conducted in Guantang, Luyi in central China from 1971-1995, were extracted and digitized. All data is going to be summarized into four parts: study site, control and measurements, malaria data (including monthly numbers of reported cases, febrile cases, parasite carriage rates, the neonatal infection rate), and entomological data regarding Anopheles sinensis.

\section{Study site}

Guantang Township is located $10 \mathrm{~km}$ south of Luyi County, $33^{\circ} 51^{\prime}$ north latitude and $115^{\circ} 21^{\prime}$ east longitude, within the river network region of Henan province, bordering Anhui Province in the east (Fig. 3). There were 114 villages in the township with a population of about 40,000 . In the early 1970 s, about $4 \%$ of the cultivated land was planted with rice. Subsequently most arable land was

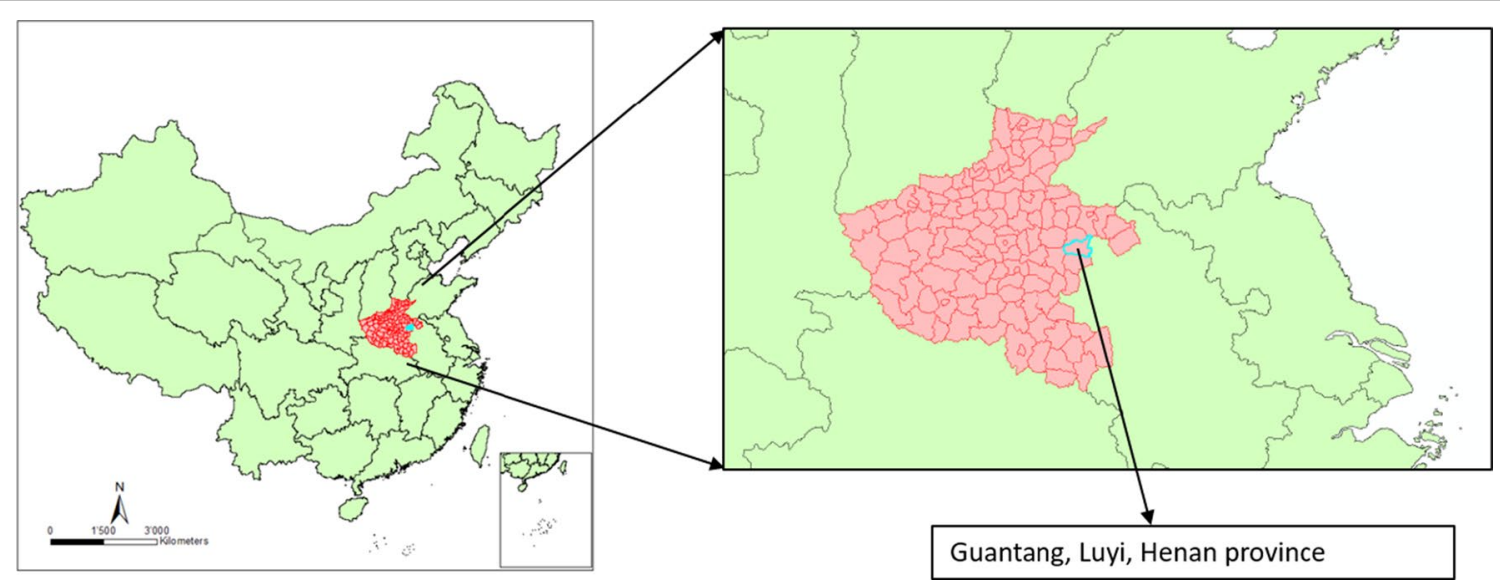

Fig. 3 Study site of Guantang, Luyi, Henan province, P. R. China: red polygons are Henan province located in central China. Blue highlighted polygon is Luyi County. Southern part of Luyi is Guantang township 
replaced with dry land crops, such as cotton. Most of the houses were poorly ventilated bungalows with wood and mud structures. In summer and early autumn, residents generally had the habit of spending the evening outdoors or sleeping outside. These villages are crossed by more than 10 small but permanent rivers with considerable aquatic vegetation. In addition, there are over 200 , mostly perennial, water bodies, of varying sizes, scattered within villages. The annual average temperature was $13.9-15{ }^{\circ} \mathrm{C}$, and the annual average precipitation was $731.8 \mathrm{~mm}$. More than $63 \%$ of the precipitation is concentrated in June-September.

In 1970, a malaria outbreak in central China (including Henan province) drew the attention of multiple levels of the Chinese administration to the problem of malaria (Fig. 1) [8]. A pilot study in Guantang was established in 1971 to study patterns of $P$. vivax transmission, and served as a demonstration project for large-scale comprehensive malaria control in central China. Investigations and research on the epidemiology and control of malaria were carried out until 1995 [4].

\section{Main control measures}

From 1971 to 1995 , the pilot site continually received special funds and implementation guidance from a professional malaria control team. Integrated control methods were implemented throughout the province. The standard control strategy was: (1) immediate treatment of diagnosed patients with 3 days chloroquine (day 1: $600 \mathrm{mg}$, day 2: $300 \mathrm{mg}$ and day 3: $300 \mathrm{mg}$ ) together with 5 or 8 days primaquine ( $150 \mathrm{mg}$ for 5 days or $180 \mathrm{mg}$ for 8 days) regimens or a 3-day-double-course therapy (chloroquine and primaquine base in divided doses); (2) in years with high incidence rates during the transmission season, mass prophylaxis with pyrimethamine was applied at the village level, while anti-malarial medication was applied in outbreak foci; (3) during the non-transmission seasons, patients with malaria infection history in the previous transmission season were treated to clear hypnozoites with different regimes at different time periods. Before mid-1970s, a regime of 8 days of primaquine ( $22.5 \mathrm{mg} /$ dose) and 2 days pyrimethamine ( $50 \mathrm{mg} / \mathrm{dose}$ ) was applied. After the mid-1970s, a regime of 3 days of chloroquine (day1: $600 \mathrm{mg}$, day 2: $300 \mathrm{mg}$ and day 3: $300 \mathrm{mg}$ ) together with 5 or 8 days of primaquine $(150 \mathrm{mg}$ for 5 days or $180 \mathrm{mg}$ for 8 days) was used. In the years with high incidence rates, a mass hypnozoite clearance programme was implemented at village level; (4) Mass anti-mosquito campaigns were carried out annually. There was no unified anti-mosquito technique applied in the province [4]. Only community-based anti-mosquito campaigns were carried out, which was one of the country's biggest "four pests"-rats, flies, mosquitoes, and sparrows campaigns introduced by Mao Zedong aiming to eradicate the transmission of pestilence and diseases [10]. In a few places with appropriate budgets, such as Guantang, larvicides, e.g. hexachlorocyclohexane powder, were applied to water bodies to eliminate mosquito larvae $[4,11]$.

\section{Malaria data collection \\ Malaria case reports}

Confirmed malaria cases were reported at different levels of the surveillance system and hospitals. Due to institutional reforms, there are differing levels of available data over the period 1971-1995. Annual malaria case data of Guantang were available for each year from 1971 to 1995, while monthly data were only available from 1971-1984.

\section{Febrile patients}

Between 1979 and 1981, febrile patients with four classified groups, namely those presumptively diagnosed as malaria by symptoms, suspected malaria, unexplained fever or suspected cold, were verified by blood smears test, the gold standard for malaria cases [12]. Due to the typical fever pattern of $P$. vivax patient, a fever that recurs every second day [12], the fever pattern was used as one criteria for vivax malaria clinical diagnosis. The blood test results of four types of patients were counted and summarized.

\section{Parasite rates of residents}

Each year from 1971 to 1982 (except the year 1981) in the months of June and November, clustered sampling, such as school-, community-based sampling, accounting for 1 to $5 \%$ of whole township population, were carried out by blood smear test (finger-prick test) to check for malaria infection (Table 1). After 1983, this sampling investigation was discontinued due to low parasite rates and difficulty in collecting large amounts of blood samples.

\section{Antibody survey}

From 1978 to 1995 (with a few years omitted), the indirect fluorescent antibody (IFA) test [13] was used to examine malaria antibody levels within randomly selected residents.

\section{Malaria infection in neonates}

From 1976 to 1981, infants born between November in previous year and October of the current year were required to undergo blood tests to identify any malaria infection if fever occurred during between beginning of the transmission season in June of the current year through to June next year (see Fig. 4). Malaria infection rates for the year were calculated given the confirmed cases in this newborn infants' cohort. Those who 
Table 1 Parasitological surveys by clustered sampling in Guantang in the months of June and November from 1971 to 1982 (except the year 1981)

\begin{tabular}{|c|c|c|c|c|}
\hline \multirow[b]{2}{*}{ Year } & \multicolumn{2}{|l|}{ June } & \multicolumn{2}{|l|}{ November } \\
\hline & $\begin{array}{l}\text { No. positive } \\
\text { cases }\end{array}$ & $\begin{array}{l}\text { No. sampled } \\
\text { people }\end{array}$ & $\begin{array}{l}\text { No. positive } \\
\text { cases }\end{array}$ & $\begin{array}{l}\text { No. } \\
\text { sampled } \\
\text { people }\end{array}$ \\
\hline 1971 & 38 & 2018 & 10 & 813 \\
\hline 1972 & 5 & 1141 & 8 & 1053 \\
\hline 1973 & 3 & 1141 & 7 & 1139 \\
\hline 1974 & 3 & 1113 & 2 & 2062 \\
\hline 1975 & 1 & 458 & 1 & 293 \\
\hline 1976 & 0 & 667 & 0 & 699 \\
\hline 1977 & 0 & 441 & 3 & 429 \\
\hline 1978 & 0 & 443 & 0 & 464 \\
\hline 1979 & 0 & 431 & 0 & 300 \\
\hline 1980 & 1 & 300 & 1 & 300 \\
\hline 1982 & 0 & 503 & 0 & 542 \\
\hline
\end{tabular}

experienced symptoms during the transmission season from June to December were recorded as short-latency episodes, while those who experience symptoms from January the following year until the next transmission season were classified as long-latency episodes.

\section{Entomological study of Anopheles sinensis}

Mosquitoes were trapped using a variety of methods, differing in trap location (indoor/outdoor), the host used as bait (human and/or cattle), time and duration [e.g. $2 \mathrm{~h}$ after sunset/overnight $(8 \mathrm{pm}-4 \mathrm{am})]$. Anopheles sinensis was the only malaria vector found in the pilot study area [11]. No other Anopheles mosquito species were detected.

\section{Human and cattle outdoor trapping for $2 \mathrm{~h}$ after sunset}

For each year between 1971 and 1984, every 10 days during the transmission season from the beginning of June until the end of October, fixed-point outdoor mosquito landing collections were carried out in parallel on both human and cattle. The two mosquito traps were separated by $100 \mathrm{~m}$ and mosquitoes were caught continuously for $2 \mathrm{~h}$ after sunset.

\section{Human indoor/outdoor overnight trapping}

Once a month in the years 1976, 1977 and 1982, from June to September, indoor human mosquito landing collections were carried out from 8 pm until 4 am and the number of mosquitoes was recorded. In addition, fixedpoint outdoor human landing collections were conducted from $8 \mathrm{pm}$ until 4am every 10 days from June until the end of October in 1975 through to 1984.

\section{Habitats of indoor resting mosquitoes}

Anopheles sinensis uses a wide range of resting places. Between 1971 and 1975, from May to October each year, female mosquitoes were caught by a person for $15 \mathrm{~min}$ inside various buildings, including bedrooms, cattle stalls, pig stalls, garages, other rooms, and brick kilns.

\section{Blood-feeding behaviour}

In 1975 and 1978, newly blood-fed female mosquitoes in different types of habitats in the wild were caught. Blood samples were tested by an electrophoresis antigen-antibody reaction to define blood-feeding hosts [14]. From June to September of 1975, female mosquitoes under the bridge Zhouqiao near Guantang village were captured to check blood-fed specimens. The stage of blood meal digestion within a mosquito was classified according to the Sella scale [4].

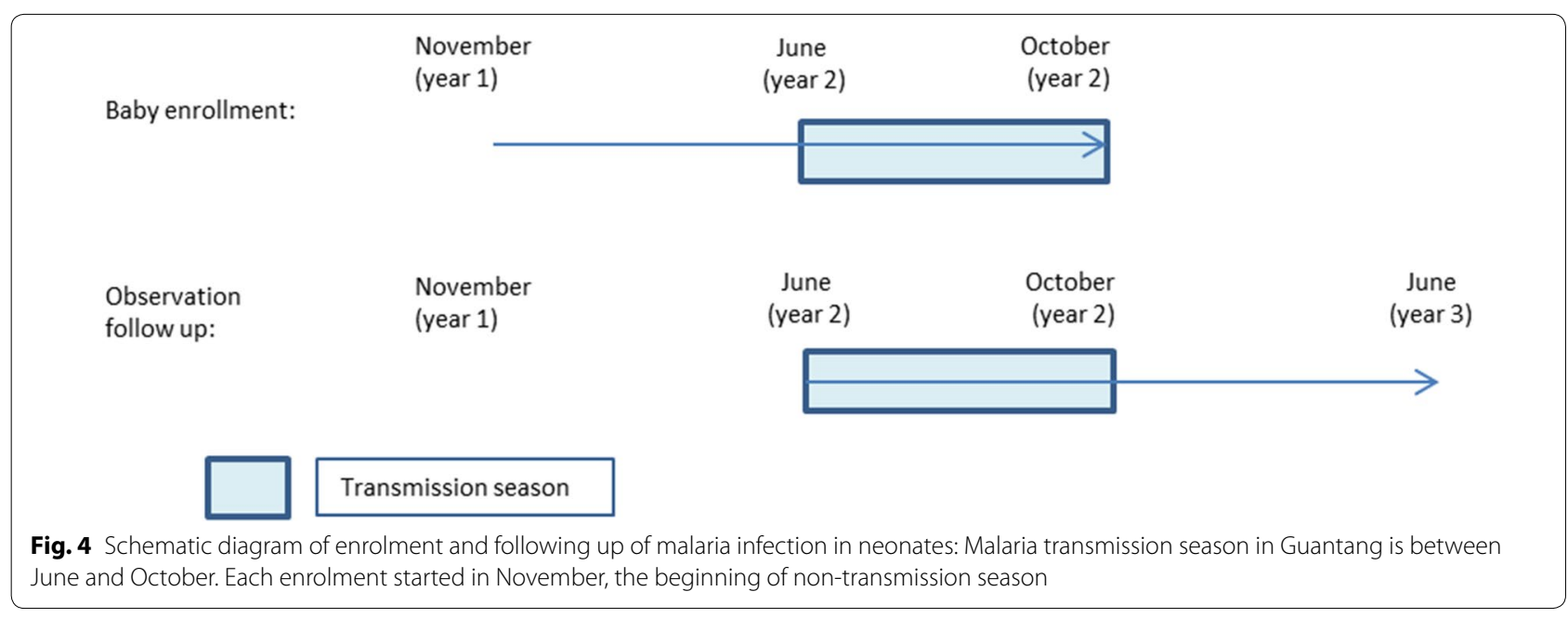




\section{Vectorial capacity}

Vectorial capacity is often used to express malaria transmission risk by local vector populations (see formular below). Between 1975-1984, vectorial capacity (C) from June to September was estimated based on the Garrett-Jones's original Eq. (1) [15] by local malaria health workers:

$$
C=\frac{m a^{2} p^{n}}{-\ln (p)}
$$

where $m$ is the ratio of mosquitoes to humans; $a$ the rate at which mosquitoes bite humans (per day) biting rates; $n$ the parasite's extrinsic incubation period (EIP, $n$ days); and $p$ is the mosquito survival through one day.

\section{Results}

Following 25 years of significant effort, the incidence of malaria in Guantang decreased from 4,333 cases per 10,000 people in 1970 before integrated implementation to $0.23 / 10,000$ in 1991 , and no cases observed between 1992-1995. There were some epidemic fluctuations from 1977 to 1981 (Fig. 5). During the study period the estimated parasite rate of residents, measured antibody levels and the estimated neonatal infection rate also decreased, indicating that the integrated malaria control measures effectively controlled malaria and achieved the goal of malaria elimination.

\section{Febrile patients}

From 1979 to 1981, parasite positive rates of febrile patients, with diagnosis of malaria by symptom, suspected malaria, unexplained fever or suspected cold, were $53.2 \%$ (666/1252), 47.4\% (704/1485), and 9.1\% (117/ $1286)$ and $13.3 \%(242 / 1822)$, respectively (See Table 2 ). Among all 1,729 malaria cases, malaria symptom-based diagnosis correctly detected only 666 cases $(38.5 \%)$. Taking into consideration microscopy as malaria diagnosis gold-standard, 61.5\% (1063/1729) malaria infections were underreported and 46.8\% (586/1252) non-malaria cases were falsely reported as malaria infections. Prior to this period, reports of malaria cases in other places of Henan province were mainly based on clinically symptomatic diagnosis.

\section{Recurrent malaria}

Malaria cases during the non-transmission seasons from 1972 to 1987 were interviewed and recorded. Most malaria cases $(78.3 \%)$ in the non-transmission season exhibited no symptoms in the previous transmission season (Table 3).

Each year from 1971 to 1984, a certain number of malaria cases in the transmission season were followed up to observe recurrent rates. Among 14,320 malaria cases followed, less than $6 \%$ had recurrent malaria during the following non-transmission season (Table 4).

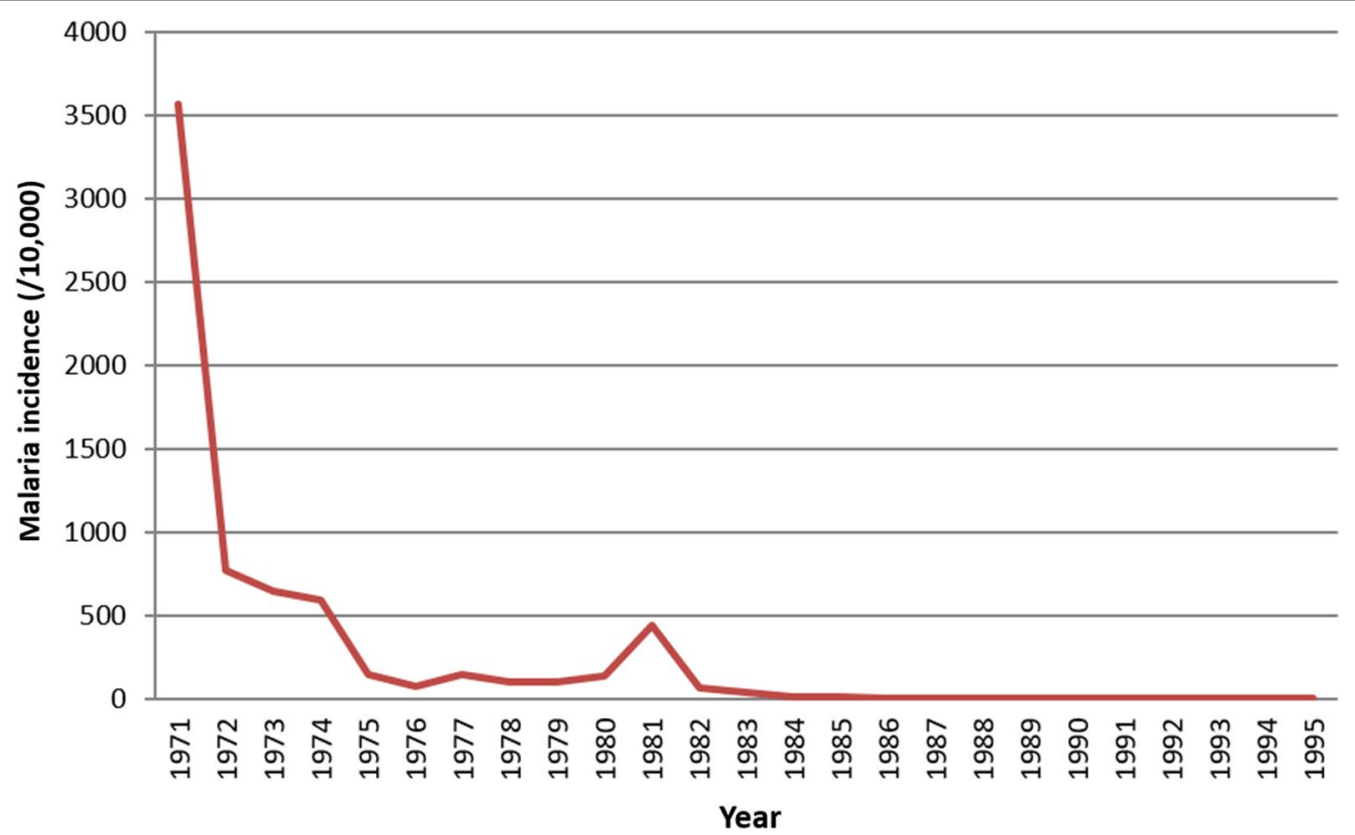

Fig. 5 Malaria incidence rate in Guantang 1971-1995. A fluctuation observed in 1981 
Table 2 Plasmodium vivax positive rates of febrile patients with diagnosis of malaria by symptom, suspected malaria, unexplained fever or suspected cold in Guantang between 1979 and 1981

\begin{tabular}{|c|c|c|c|c|c|}
\hline & \multicolumn{4}{|c|}{ Febrile patient groups } & \multirow[b]{2}{*}{ Total } \\
\hline & $\begin{array}{l}\text { Malaria clinical } \\
\text { symptom }\end{array}$ & Suspected malaria & Unexplained fever & Suspected cold & \\
\hline No. cases & 1252 & 1485 & 1286 & 1822 & 5845 \\
\hline No. confirmed by microscope & 666 & 704 & 117 & 242 & 1729 \\
\hline Parasite positive rates & $53.2 \%$ & $47.4 \%$ & $9.1 \%$ & $13.3 \%$ & \\
\hline
\end{tabular}

Table 3 Malaria cases investigation in non-transmission season in Guantang between 1971 and 1987

\begin{tabular}{llcl}
\hline Year & $\begin{array}{l}\text { No. cases in non- } \\
\text { transmission } \\
\text { season }\end{array}$ & $\begin{array}{l}\text { Without malaria symptom } \\
\text { in previous transmission } \\
\text { season }\end{array}$ & Ratio (\%) \\
\hline 1972 & 540 & 227 & 42.04 \\
1973 & 663 & 510 & 76.92 \\
1974 & 705 & 676 & 95.89 \\
1975 & 160 & 127 & 79.38 \\
1976 & 163 & 151 & 92.64 \\
1977 & 45 & 43 & 95.56 \\
1978 & 112 & 102 & 91.07 \\
1979 & 100 & 85 & 85.00 \\
1980 & 134 & 111 & 82.84 \\
1981 & 241 & 198 & 82.16 \\
1982 & 64 & 50 & 78.13 \\
1983 & 49 & 44 & 89.80 \\
1984 & 19 & 19 & 100.00 \\
1985 & 3 & 3 & 100.00 \\
1986 & 0 & 0 & - \\
1987 & 1 & 1 & 100.00 \\
Total & 2999 & 2347 & 78.3 \\
\hline
\end{tabular}

\section{Parasite carriage}

The carriage rate of asexual blood-stage parasite in residents in the beginning and end of transmission season were very similar. Although parasite carriage fluctuated between years, overall it declined from $1.7 \%$ in 1971 to 0 in the 1980 s. Of the 7,634 clinically symptomatic patients who were positively confirmed by blood smear tests between 1977 and 1982, gametocytes were detected in 5,251 (68.8\%). In addition, among the 59 confirmed cases with first clinical episodes, gametocytes were detected in 55 on the first day of symptoms. The carriage rate of gametocytes was high during this period.
Table 4 Recurrent rate of malaria cases in transmission season in Guantang between 1971 and 1984

\begin{tabular}{lccc}
\hline Year & $\begin{array}{l}\text { No. malaria } \\
\text { in transmission } \\
\text { season }\end{array}$ & $\begin{array}{l}\text { No. recurrent cases } \\
\text { in non-transmission } \\
\text { season }\end{array}$ & Ratio (\%) \\
\hline 1971 & 5804 & 90 & 1.55 \\
1972 & 2200 & 313 & 14.23 \\
1973 & 1654 & 153 & 9.25 \\
1974 & 1498 & 29 & 1.94 \\
1975 & 400 & 33 & 8.25 \\
1976 & 89 & 12 & 13.48 \\
1977 & 506 & 2 & 0.40 \\
1978 & 270 & 10 & 3.70 \\
1979 & 297 & 15 & 5.05 \\
1980 & 396 & 23 & 5.81 \\
1981 & 899 & 43 & 4.78 \\
1982 & 177 & 14 & 7.91 \\
1983 & 107 & 5 & 4.67 \\
1984 & 23 & 0 & 0.00 \\
Total & 14,320 & 742 & 5.18 \\
\hline
\end{tabular}

\section{Antibody survey}

According to the documented reports, the antibody positivity was higher before 1984 . Since 1985 , about $1 \%$ of the population were positive for malaria antibodies by IFA (Table 5 ).

\section{Malaria infection in neonates}

From 1976 to 1981, among 4,858 babies examined for malaria infection, 41 malaria cases were confirmed by blood smear test of which 25 cases occurred between July to December of the year enrolled, and 13 cases in the non-transmission period of the following year (See Table 6). The ratio of long-term to short-term incubation is therefore 1:2.15.

\section{Entomological characteristics of Anopheles sinensis Seasonal density}

The number of mosquitoes trapped by either outdoor human and cattle traps between years 1971 and 1984 
Table 5 Malaria antibody levels within randomly selected residents in Guantang between 1978 and 1995 the indirect fluorescent antibody (IFA) test (except years 1981-1983 and 1987)

\begin{tabular}{llll}
\hline Year & $\begin{array}{l}\text { No. people } \\
\text { tested }\end{array}$ & $\begin{array}{l}\text { No. people } \\
\text { positive }\end{array}$ & Positive rate (\%) \\
\hline 1978 & 907 & 80 & 8.82 \\
1979 & 611 & 51 & 8.35 \\
1980 & 524 & 72 & 13.74 \\
1984 & 269 & 11 & 4.09 \\
1985 & 300 & 1 & 0.33 \\
1986 & 627 & 2 & 0.32 \\
1988 & 400 & 0 & 0 \\
1989 & 282 & 0 & 0 \\
1990 & 300 & 0 & 0 \\
1991 & 400 & 5 & 1.25 \\
1992 & 331 & 3 & 0.91 \\
1993 & 845 & 8 & 0.95 \\
1994 & 862 & 6 & 0.70 \\
1995 & 393 & 6 & 1.53 \\
\hline
\end{tabular}

indicated that the density of mosquitoes was likely very low in late May or early June, with the first density peak in late June, and a second one in late August. The density dropped sharply in September and was too low to measure precisely in October (Fig. 6). Other observations also showed that development of the ovaries of female mosquitoes ceased in late November $[4,11]$.

\section{Blood-feeding behaviour}

Host preferences: When searching for blood hosts, An. sinensis expresses a preference for cattle, with an estimated average ratio of 1:11.9 between human and cattle in paired landing catches (Fig. 6). Most of the indoor resting mosquitoes were captured in cattle and pig stalls.

Indoor/outdoor preference: The ratio of number of mosquitoes captured by human indoor / outdoor overnight landing collections was 1:7.73, indicating that An. sinensis prefers outdoor blood-feeding (Table 7).

Blood-feeding activity overnight: Blood-feeding activities of An. sinensis persist throughout the night. Although there were some variations between different months, generally two blood-feeding peaks can be detected. One was around $8 \mathrm{pm}, 2 \mathrm{~h}$ after sunset. The other one happens between $11 \mathrm{pm}$ and $2 \mathrm{am}$ (Table 8 ).

Human blood index: In 1975 and 1978, 170 and 433 newly blood-fed female mosquitoes in different types of habitats in the wild were caught. Of these, 44 and 132 were human blood positive in 1975 and 1978, respectively, corresponding to an average human blood index of 0.29 .

After blood-feeding: Among the 523 An. sinensis captured under the bridge in Zhouqiao, the gastric blood of Sella scale II-III, accounted for $30.8 \%$, and IV-V scale accounted for $38.8 \%$.

Sporozoite rate: From June to August in 1974, a total of 2,068 An. sinensis were dissected. Only one mosquito's salivary gland was positive for sporozoites corresponding to a natural infection rate of $0.05 \%$.

Vectorial capacity: Between 1975 to 1984, a substantial fluctuation in vectorial capacity was observed with peaks in 1977 and 1979. After 1980, vectorial capacity showed a continuously declining trend (Fig. 7).

\section{Discussion}

Plasmodium vivax was the major malaria species in Henan province and responsible for more than $90 \%$ of the local malaria [3]. Transmission was unstable with several outbreaks in 1950s, 1960s and 1970s. From the 1980s onwards, although the incidence rate was low, there was considerable inter-annual variation especially between 1985 and 2009 [16]. Henan Province, and Guantang in particular, is located in the warm temperate zone with a clear seasonal pattern of transmission, with the transmission season usually spanning June to October. From November onwards, low ambient temperatures

Table 6 Incubation period study of primary infection among neonates malaria in Guantang between 1976 and 1981

\begin{tabular}{|c|c|c|c|c|c|c|c|c|c|c|c|c|c|c|}
\hline \multirow[t]{2}{*}{ Year } & \multirow{2}{*}{$\begin{array}{l}\text { No. total } \\
\text { enrolled } \\
\text { neonates }\end{array}$} & \multirow[t]{2}{*}{ No. total cases } & \multicolumn{12}{|c|}{ Month } \\
\hline & & & 7 & 8 & 9 & 10 & 11 & 12 & 1 & 2 & 3 & 4 & 5 & 6 \\
\hline 1976 & 750 & 1 & & & & & 1 & & & & & & & \\
\hline 1977 & 917 & 6 & & 2 & 1 & & & & & & & & 1 & 2 \\
\hline 1978 & 1014 & 5 & & & 1 & 4 & & & & & & & & \\
\hline 1979 & 909 & 6 & & 3 & 2 & & & & & & 1 & & & \\
\hline 1980 & 664 & 16 & & 1 & 1 & 3 & 2 & & & & 3 & & 5 & 1 \\
\hline 1981 & 604 & 7 & 3 & 2 & 2 & & & & & & & & & \\
\hline Total & 4858 & 41 & 3 & 8 & 7 & 7 & 3 & & & & 4 & & 6 & 3 \\
\hline
\end{tabular}




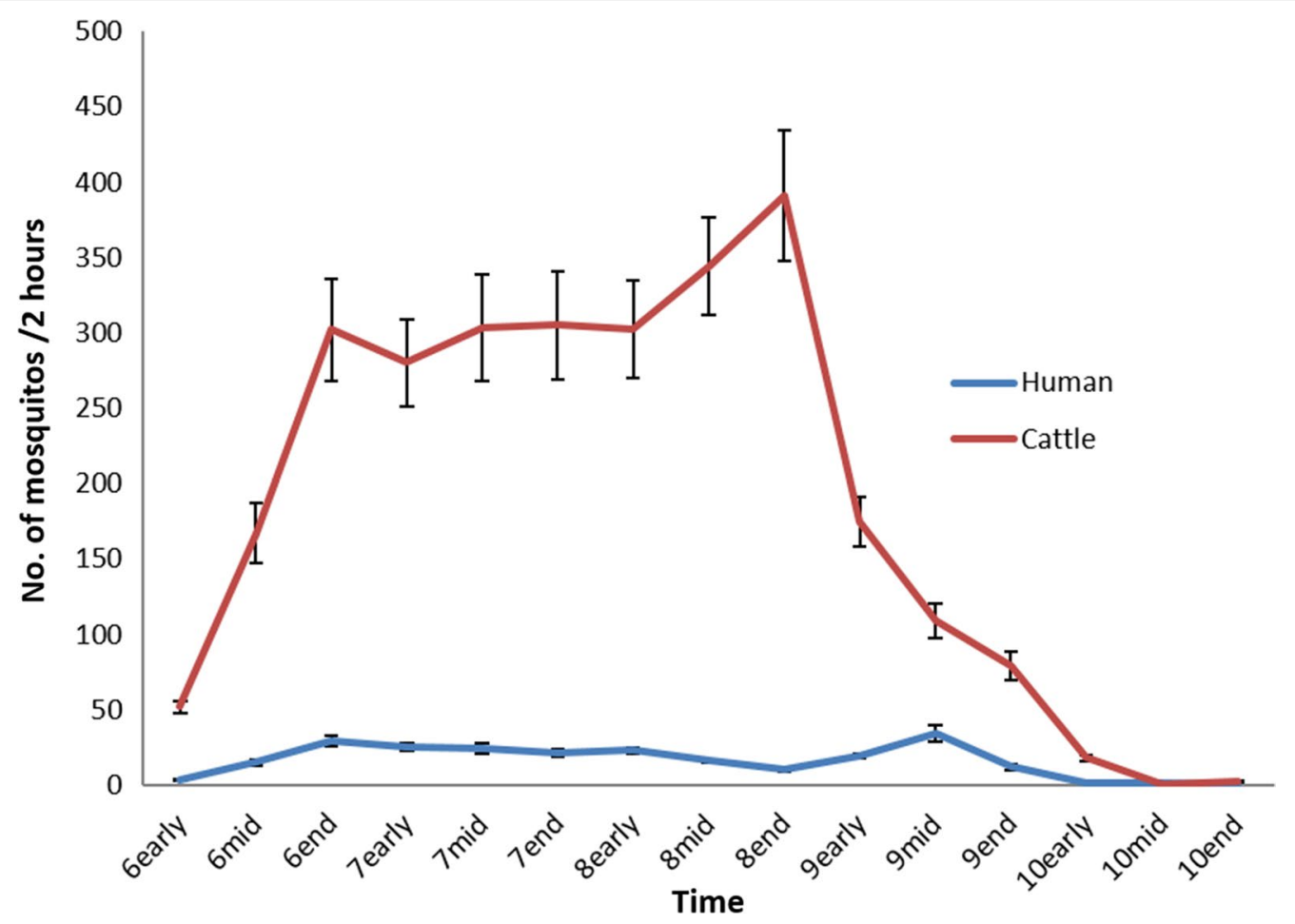

Fig. 6 Fixed-point outdoor mosquito landing collections on both human and cattle by every 10 days during the transmission season from the beginning of June until the end of October: solid line is the averaged number of mosquitos between 1971 and 1984 with 95\% confidence interval

Table 7 Number of mosquitos captured by human indoor/outdoor overnight landing collections in different months

\begin{tabular}{|c|c|c|c|c|c|c|c|c|}
\hline \multirow[t]{2}{*}{ Year } & \multicolumn{2}{|l|}{ June } & \multicolumn{2}{|l|}{ July } & \multicolumn{2}{|l|}{ August } & \multicolumn{2}{|c|}{ September } \\
\hline & Indoor & Outdoor & Indoor & Outdoor & Indoor & Outdoor & Indoor & Outdoor \\
\hline 1976 & 3 & 13 & 6 & 44 & 3 & 11 & 0 & 1 \\
\hline 1977 & 1 & 9 & 3 & 60 & 4 & 16 & 1 & 10 \\
\hline 1982 & 0 & 1 & 0 & 0 & 1 & 2 & 0 & 4 \\
\hline
\end{tabular}

inhibited mosquito movement and survival, preventing transmission. However, due to global warming, the temporal limits of mosquito survival have expanded, prolonging the potential malaria transmission season [17]. The central part China (i.e., Anhui, Henan and Jiangsu provinces) form a climate sensitive zone [17] that experienced re-emergence of malaria between 2000 and 2006 and the surveillance response system needs to continue to be consolidated in this specific region $[1,18]$.

The malaria parasite phenotype in Guantang was found to be similar to that of $P$. vivax described as North Korean [19], but the Guantang analysis indicates the primary episode and latency of over 12 months is relatively longer than $P$. vivax North Korean strains, with relapses occuring roughly 8-9 months after primary infection [19]. Experiments conducted by Le-Yuan Shang found that incubation periods of $P$. vivax in Henan Province could be up to 443 days long ( 15 months) (238-443 days) [4]. Among the malaria cases in Henan from January to May (non-transmission season) in 1972-1987, those without malaria symptoms in the previous year accounted for more than $76.9 \%(2,347 / 2,999)$ which indicates that a long incubation period (latency) malaria was dominant in the non-transmission period.

A study carried out by Coatney suggests that the main factor determining duration of latency or the inter relapse interval (for the first relapses with genetically homologous parasites) was inoculum size of sporozoites rather than immunity [20]. A further study conducted by Le-Yuan Shang confirmed that Henan Province had predominately long incubation type $P$. vivax (temperate) with a ratio of long- to short-term incubation infections in neonates of $1: 2.15$, similar to the outcomes of infections with a high inoculum size in adults [4]. 
Table 8 Mosquito blood-feeding activity over night from $8 \mathrm{pm}$ to 3 am by indoor human mosquito landing collections from June to September: the number of mosquitos presented in each cell is the averaged value between 1975 and 1984

\begin{tabular}{llllc}
\hline \multirow{5}{*}{ Time } & \multicolumn{4}{l}{ No. mosquitos/person.night } \\
\cline { 2 - 5 } & June & July & Aug & Sept \\
\hline 8:00 pm & 11 & 20 & 13 & 17 \\
9:00 pm & 11 & 13 & 11 & 11 \\
10:00 pm & 3 & 18 & 11 & 10 \\
$11: 00 \mathrm{pm}$ & 13 & 20 & 12 & 7 \\
12:00 pm & 26 & 22 & 14 & 3 \\
1:00 pm & 15 & 24 & 20 & 6 \\
2:00 pm & 8 & 26 & 12 & 10 \\
3:00 pm & 6 & 13 & 7 & 4 \\
4:00 pm & 3 & 14 & 4 & 5 \\
\hline
\end{tabular}

There is no original data available for each year

Owing to the long latency in Henan, clearing hypnozoites in asymptomatic hosts by mass treatment with primaquine was very likely a driver and thus of considerable importance in reducing the reservoir of infection, which would otherwise have been the main contributor in the following transmission season. This finding provided a scientific basis for the implementation of mass campaigns of liver stage hypnozoite clearance in Henan province especially in the years that experienced malaria outbreaks, such as 1960s and 1970s. However, in low-endemic areas this strategy may not be cost-effective, since mass treatment in non-transmission seasons to support hypnozoite elimination entails targeting a large number of people, a large demand for drugs, and enormous resources in terms of manpower, all of which requires in-depth organization.

There is the additional risk of clustered adverse reactions [21], and the approach is ethically controversial if only a small number of the population are carrying hypnozoites or at risk. Therefore, $\mathrm{He}$ et al. suggested an alternative strategy to mass treatment that a) in a village with incidence rate of $>3 \%$, positive episodes and the residents within $50 \mathrm{~m}$ radius take a 3-day- chloroquine and a 5 or 8-day-primaquine regimen treatment; b) in a village with incidence rate of $<3 \%$, only positive episodes receive a 3-day-chloroquine and a 5 or 8-day-primaquine regimens; c) during non-transmission seasons a 4-day-chloroquine and primaquine treatment could be given, but only targeted on people with malaria infection history [21]. When scholars in China found that $86 \%$ of malaria cases are distributed within $100 \mathrm{~m}$ of the main water body [22], it was proposed that the scope of the

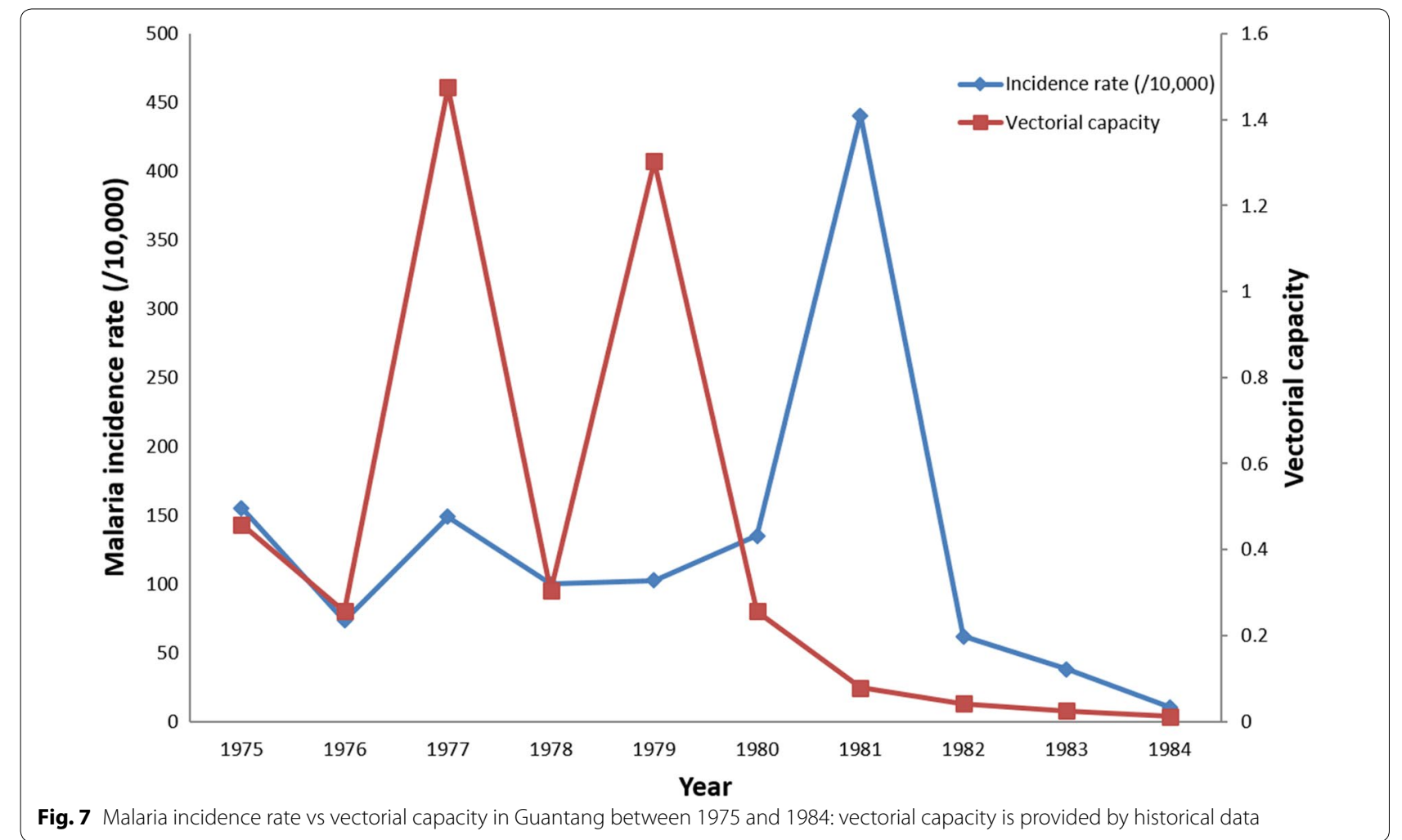


hypnozoite clearance programme should be defined by location of detection, the distance to water body and the living conditions of neighbourhood residents. This strategy was assessed in a pilot study in Anhui Province with significant success [22].

Episodes of recurrent parasitaemia following treatment may be due to recrudescence of the initial infection, reflecting failure of the drug to clear the infection, or may be due to re-infection, or due to relapse by activated hypnozoites. Since the causes in Henan were not distinguishable with available data, only episodes in the nontransmission season could be distinguished. For most years, malaria episodes in the non-transmission season with documented history of malaria accounted for less than $10 \%$ of total episodes in the previous transmission season, with an average recurrent rate of $5.18 \%$ (Table 4). Thus a 3-day-chloroquine $(1200 \mathrm{mg})$ and 5 or 8-dayprimaquine (150 or $180 \mathrm{mg}$ ) administration efficiently control the recurrence rate of malaria in the pilot area. Assuming complete clearance of parasites in the blood by chloroquine, $5.18 \%$ cases were still attributed to drug resistance to primaquine. This is slightly higher than the report of $4.5 \%$ by Saifi with administration with $1500 \mathrm{mg}$ chloroquine and $75 \mathrm{mg}$ primaquine [2].

The average HBI of outdoor mosquitoes in Guantang in $1970 \mathrm{~s}$ was 0.29 , indicating that An. sinensis is an animal preferring species, similar to findings from a study carried out in Nanjing, Jiangsu Province in the period of $1952-1955$ with HBI 0.26 by testing $2616 A n$. sinensis [14]. Field investigations in northern Anhui in August and September, 2005 showed that HBI of $A n$. sinensis was 0.18 [18, 23]. Studies carried out in 4 counties in Yunnan Province between June and August, 2012 detected low HBI of 0.018 [24]. There is thus large variation in the estimates of HBI obtained by these different surveys. However, a clear declining trend in time of the estimated HBI corresponds to a period of economic growth and improved rural living conditions such as mosquito meshes installed in doors and windows and popularity of electric fans and air conditioning. The habit of sleeping outdoors in summer has also changed. In Guantang, the An. sinensis favoured habitats such as rice fields have been gradually modified for cultivation to dry land products, such as cotton. This agricultural change has undoubtably significantly reduced contact between mosquitoes and people. The Sella scale is the indicator of mosquito gastric blood digestion degree with a lower Sella scale indicating shorter time after blood-feeding. In Guantang, around $1 / 3$ of mosquitoes were at the Sella scale II-III, which means An. sinensis belongs to the outdoor-resting mosquito species. After blood-feeding on various hosts, mosquitoes were flying to the outside of the village for oviposition.

\section{New challenges}

In areas of China with unstable transmission, epidemics on a 10 year periodicity were observed in the early 1950s, 1960s, 1970s, and 1980s [1]. These cycles have been attributed to either a regular pattern of solar activity cycle which may have an impact on regional climate and weather; or to intrinsic cycles in the dynamics of $P$. vivax malaria resulting from activation of long dormancy hypnozoites [21]. The latter hypothesis is more consistent with the finding that the outbreak in 1981 was not associated with any increase in vectorial capacity (Fig. 7). Challenges remain in the maintenance of non-transmission status owing to the long dormancy of liver stage hypnozoites.

If ecological conditions remain unchanged and Anopheles mosquitoes remain widespread, once intervention activities cease there is always potential for malaria to be re-introduced and resurge. As recently as 2015, officials reported two local cases of $P$. vivax [25] in Liaoning, where malaria transmission had been interrupted for 4 years. In recent years, the number of recorded imported falciparum malaria cases has increased with most patients being residents returning from business, tourism or work in malaria highly endemic regions, i.e. Africa and Southeast Asia [7, 26]. To mitigate risks of reintroduction, the Henan Provincial Health Department in conjunction with other relevant public bodies, continue to conduct malaria surveillance and response activities involving tracking malaria cases, screening key populations and communicating related information to control imported $P$. falciparum malaria [16].

\section{Conclusion}

The integrated malaria control approach in Guantang effectively controlled malaria and achieved elimination. Analysis of the effectiveness of the programme can provide guidance to other regions or countries with similar ecological settings aiming to move from malaria control to elimination. There is a potential challenge in the maintenance of non-transmission status owing to imported cases and the long dormancy of liver stage hypnozoites.

\section{Acknowledgements \\ We acknowledge the contributions of all the anonymous people who worked for the malaria control and elimination programme and also the people who helped with the digitization.}

\section{Authors' contributions}

GJY conceived the paper, collated data, analysed results and wrote the first version of the manuscript. LY, HWZ, LYS, XNZ supported data collection, collation and interpreting results. TAS and MAP supported interpretation of results and revision of the manuscript. All authors read and approved the final manuscript. 


\section{Funding}

GJY is grateful to the National Nature Science Foundation (grant no. 81573261). MAP was funded under Swiss National Science Foundation PP00P3_170702. TAS was funded under Bill \& Melinda Gates Foundation grant OPP 1032350.

\section{Ethics approval and consent to participate}

There is no ethics issues in this study.

\section{Consent for publication}

All authors assurances that the manuscript a) is an original work, b) has not been previously published whole or in part, and c) is not under consideration for publication elsewhere.

\section{Competing interests}

The authors declare that there are no competing interests.

\section{Author details}

${ }^{1}$ Swiss Tropical and Public Health Institute, Socinstrasse 57, 4002 Basel, Switzerland. ${ }^{2}$ University of Basel, Basel, Switzerland. ${ }^{3}$ Laboratory of Tropical Environment and Health, Hainan Medical University, Haikou, Hainan, People's Republic of China. ${ }^{4}$ Henan Center for Disease Control and Prevention, Zhengzhou, Henan, People's Republic of China. ${ }^{5}$ Chinese Center for Disease Control and Prevention, National Institute of Parasitic Diseases, Shanghai, People's Republic of China. ${ }^{6}$ Key Laboratory of Parasite and Vector Biology, WHO Collaborating Center for Tropical Diseases, Ministry of Health, Shanghai, People's Republic of China.

Received: 11 February 2020 Accepted: 15 November 2020 Published online: 23 November 2020

\section{References}

1. Su SZ, Ma YX, Wang Z. Malaria control and research in Henan province. Zhengzhou: Zhong Yuan Farmer Publication; 1995.

2. Saifi MA, Wajihullah SMI, AI-Khalifa MS. Malaria: patterns of relapse and resistance. J King Saud Univ Sci. 2010;22:31-6.

3. Xu B, Su Y, Liu Y. Malaria evaluation on the control effect in Henan Province during 1970-2010 (in Chinese). Henan J Prev Med. 2011;22:321-6.

4. Shang $L Y, X u$ BL, Su YP. Malaria endemic and control in Henan province. 1st ed. Zhengzhou: Zhongyuan Publishing Media Group; 2011.

5. Wang LP. Systematic review: national notifiable infectious disease surveillance system in China. Online J Public Health Inform. 2019:11:e414.

6. WHO. Our Work in China. Securing a malaria-free future for China. [cited 2019; Available from: http://www.wpro.who.int/china/mediacentre/facts heets/infectious_diseases/en/.

7. Feng J, Zhang L, Huang F, Yin JH, Tu H, Xia ZG, et al. Ready for malaria elimination: zero indigenous case reported in the People's Republic of China. Malar J. 2018;17:315.

8. Feng J, Xiao $\mathrm{H}$, Zhang $\mathrm{L}$, Yan $\mathrm{H}$, Feng $\mathrm{X}$, et al. The Plasmodium vivax in China: decreased in local cases but increased imported cases from Southeast Asia and Africa. Sci Rep. 2015:5:8847.
9. Zhang L. Malaria situation in the People's Republic of China in 2016 (in Chinese). Chin J Parasitol Parasit Dis. 2017;35:5.

10. Wikipedia. Four Pests Campaign [cited 20192019 October 30]. https:// en.wikipedia.org/wiki/Four_Pests_Campaign.

11. Li P, Shang LY, Zhuang JA. Mosquito vector survey in Henan province (in Chinese). J Pract Parasit Dis. 2001;9:2.

12. Institute of Parasitic Diseases, Chinese Academy of Medical Sciences. Practical malaria, 1st ed. Beijing: People's Medical Publishing House; 1978.

13. Duo-Quan W, Lin-Hua T, Zhen-Cheng G, et al. Application of the indirect fluorescent antibody assay in the study of malaria infection in the Yangtze River Three Gorges Reservoir. China Malar J. 2009;8:199.

14. Li JL, Luo GX (1985) Investigation on the blood sucking behaviour of Anopheles mosquitoes in Jiangsu. JPD 200-1

15. Garrett-Jones $C$. The human blood index of malaria vectors in relation to epidemiological assessment. Bull World Health Organ. 1964;30:241-61.

16. Zhang L, Feng J, Zhang SS, Xia ZG, Zhou SS. The progress of national malaria elimination and epidemiological characteristics of malaria in China in 2017. Chin J Parasitol Parasit Dis. 2018;36:201-10.

17. Yang GJ, Tanner M, Utzinger J, Malone JB, Bergquist R, Chan EY, et al. Malaria surveillance-response strategies in different transmission zones of the People's Republic of China: preparing for climate change. Malar J. 2012;11:426.

18. Shen MZ. Investigation of malaria transmission risk factors in Anhui province (in Chinese). Chinese J Pathog Biol. 2006;1:3.

19. White NJ. Determinants of relapse periodicity in Plasmodium vivax malaria. Malar J. 2011;10:297.

20. Coatney GR, Cooper WC, Young MD. Studies in human malaria. XXX. A summary of 204 sporozoite-induced infections with the Chesson strain of Plasmodium vivax. J Natl Malar Soc. 1950;9:381-96.

21. He B. The distribution of long incubation P. vivax in China and the proportion of long/short incucation (in Chinese). Chinese Public Health. 1986;5:3.

22. Zhou SS, Zhang SS, Wang JJ, Zheng X, Huang F, Li WD, et al. Spatial correlation between malaria cases and water-bodies in Anopheles sinensis dominated areas of Huang-Huai plain. China Parasit Vectors. 2012;5:106.

23. Fang-Hong Y. Study on threshold density of Anopheles sinensis for transmission of malaria in the Northern Anhui Province. J Trop Med. 2007;7:3.

24. Zhang JW, Jiang JW, Zeng XC. [Study of ecological habits of important malaria vectors in epidemic-prone regions of malaria in Yunnan province, China](in Chinese). Chin J Vector Biol Control. 2015;26:4.

25. Sun YW, Yu DM, Chen J. Two individual incidences of vivax malaria in Dandong municipality of Liaoning province (in Chinese). Chin J Public Health. 2017;33:3.

26. Xian Y. Analysis on the evolution and trend of malaria prevention and control policies in China (in Chinese). Chin J Health Policy. 2017;10:5.

\section{Publisher's Note}

Springer Nature remains neutral with regard to jurisdictional claims in published maps and institutional affiliations.

\footnotetext{
Ready to submit your research? Choose BMC and benefit from:

- fast, convenient online submission

- thorough peer review by experienced researchers in your field

- rapid publication on acceptance

- support for research data, including large and complex data types

- gold Open Access which fosters wider collaboration and increased citations

- maximum visibility for your research: over $100 \mathrm{M}$ website views per year
}

At BMC, research is always in progress.

Learn more biomedcentral.com/submissions 